\title{
Photobiomodulation therapy by NIR laser in persistent pain: an analytical study in the rat
}

\author{
Laura Micheli ${ }^{1}$ - Lorenzo Di Cesare Mannelli ${ }^{1}$ Elena Lucarini $^{1} \cdot$ Francesca Cialdai $^{2}$. \\ Leonardo Vignali $^{2} \cdot$ Carla Ghelardini $^{1} \cdot$ Monica Monici $^{2}$
}

Received: 1 March 2017 / Accepted: 5 July 2017 / Published online: 28 July 2017

(C) Springer-Verlag London Ltd. 2017

\begin{abstract}
Over the past three decades, physicians have used laser sources for the management of different pain conditions obtaining controversial results that call for further investigations. In order to evaluate the pain relieving possibilities of photobiomodulation therapy (PBMT), we tested two near infrared (NIR) laser systems, with different power, against various kinds of persistent hyperalgesia animal models. In rats, articular pain was reproduced by the intra-articular injection of sodium monoiodoacetate (MIA) and complete Freund's adjuvant (CFA), while compressive neuropathy was modelled by the chronic constriction injury of the sciatic nerve (CCI). In MIA and CFA models, (NIR) laser (MLS-Mphi, ASA S.r.l., Vicenza, Italy) application was started 14 days after injury and was performed once a day for a total of 13 applications. In MIA-treated animals, the anti-hyperalgesic effect of laser began 5 min after treatment and vanished after $60 \mathrm{~min}$. The subsequent applications evoked similar effects. In CFA-treated rats, laser efficacy started $5 \mathrm{~min}$ after treatment and disappeared after $180 \mathrm{~min}$. In rats that underwent CCI, two treatment protocols with similar fluence but different power output were tested using a new experimental device called Multiwave Locked System laser (MLS-HPP). Treatments began 7 days after injury and were performed during 3 weeks for a total of 10 applications. Both
\end{abstract}

Lorenzo Di Cesare Mannelli

lorenzo.mannelli@unifi.it

1 Department of Neuroscience, Psychology, Drug Research and Child Health-NEUROFARBA-Pharmacology and Toxicology Section, University of Florence, VialePieraccini 6, 50139 Florence, Italy

2 ASA Campus Joint Laboratory, ASA Res. Division-Department of Experimental and Clinical Biomedical Sciences "Mario Serio", University of Florence, Florence, Italy protocols reduced mechanical hyperalgesia and hindlimb weight bearing alterations until $60 \mathrm{~min}$ after treatment with a higher efficacy recorded for the animals treated using the higher power output. In conclusion, this study supports laser therapy as a potential treatment for immediate relief of chronic articular or neuropathic pain.

Keywords NIR laser $\cdot$ PBMT $\cdot$ Osteoarthritis $\cdot$ Rheumatoid arthritis $\cdot$ Mononeuropathy $\cdot$ CFA $\cdot$ MIA
Abbreviations
MIA Monoiodoacetate
CFA Complete Freund's adjuvant
CCI Chronic constriction injury
NIR Near infrared
PBMT Photobiomodulation therapy

\section{Introduction}

Chronic pain is a complex, severe and debilitating condition that can lead to a considerable reduction in function and quality of life [1-3]. Indeed, restriction in daily activities, social life and working assets, sleep disturbances, anxiety and depression is frequently reported. The burden on society is high and includes considerable healthcare and welfare costs, as well as indirect costs such as loss of productivity and skills due to absenteeism and early retirement [4]. Patients may present with different forms of chronic pain resulting from a number of identifiable causes, including pain due to lesion or dysfunction of the nerves, spinal cord or brain (neuropathic pain) or persistent pain caused by other non-malignant conditions, such as low-back pain or pain due to inflammation of various arthritic conditions. For these reasons, the pain management is often complex and clinically 
challenging, requiring a multimodal treatment approach [5]. For the pharmacological treatment of the non-neuropathic chronic pain, opioids are an established standard. However, they are associated with significant side effects that might influence adherence to treatment or results in treatment discontinuation [6]. Furthermore, the pharmacotherapy for neuropathic pain is disappointing. Patients with neuropathy do not respond to nonsteroidal anti-inflammatory drugs and resistance or insensitivity to opioids is common. Patients are usually treated with tricyclic or serotonin and norepinephrine uptake inhibitors, antidepressants and anti-convulsants that all have limited efficacy and undesirable side effects. Indeed, epidemiological surveys have shown that many patients with neuropathic pain do not receive appropriate treatment $[7,8]$. Thus, the development of new effective therapies, with lower side effects, is required to effectively manage neuropathic and chronic pain.

Since many years, laser sources have been applied for the treatment of various types of pain [9-11]. This kind of photobiomodulation therapy (PBMT) has become increasingly popular because it is non-invasive, painless and no significant side effects have been reported. Research on the mechanisms underlying the analgesic effects of laser radiation is carried out since more than 30 years. However, these mechanisms are not yet fully known and the complexity in understanding them arises in part by a lack of methodological standardization of certain studies and by the many different treatment parameters and conditions used.

The purpose of the present study was to evaluate the effectiveness of high power, dual wavelength near infrared (NIR) laser sources in inducing anti-hypersensitivity effects in vivo in rat models of pain induced by different causes. Unilateral sciatic nerve chronic constriction injury (CCI) was adopted as a rat model of neuropathic pain while intra-articular injections of monoiodoacetate (MIA) and complete Freud's adjuvant (CFA) were adopted as a model of osteoarthritis and rheumatoid arthritis, respectively.

\section{Materials and methods}

\section{Animals}

For all the experiments described below, male Sprague-Dawley rats (Envigo, Varese, Italy) weighing approximately 200-250 g at the beginning of the experimental procedure were used. Animals were housed in CeSAL (Centro Stabulazione Animali da Laboratorio, University of Florence) and used 1 week after their arrival. Four rats were housed per cage (size $26 \times 41 \mathrm{~cm}^{2}$ ), fed with standard laboratory diet and tap water ad libitum, kept at $23 \pm 1{ }^{\circ} \mathrm{C}$ with a 12 -h light/dark cycle and light at 7 a.m. All animal manipulations were carried out according to the Directive 2010/63/EU of the European parliament and of the European Union council (22 September 2010) on the protection of animals used for scientific purposes. The ethical policy of the University of Florence complies with the Guide for the Care and Use of Laboratory Animals of the US National Institutes of Health (NIH Publication No. 85-23, revised 1996; University of Florence assurance number: A5278-01). Formal approval to conduct the experiments described was obtained from the Italian Ministry of Health (No. 54/2014-B) and from the Animal Subjects Review Board of the University of Florence. Experiments involving animals have been reported according to ARRIVE guidelines [12]. All efforts were made to minimize animal suffering and to reduce the number of animals used.

\section{MIA-induced osteoarthritis}

Unilateral osteoarthritis was induced by injection of MIA(Sigma-Aldrich) into the tibiotarsal joint $[13,14]$. Rats were slightly anaesthetized by $2 \%$ isoflurane, the left leg skin was sterilized with $75 \%$ ethyl alcohol, and the lateral malleolus located by palpation; then, a 28 -gauge needle was inserted vertically to penetrate the skin and turned distally for insertion into the articular cavity at the gap between the tibiofibular and tarsal bone until a distinct loss of resistance was felt. Two milligrams of MIA in $25 \mu \mathrm{L}$ saline was delivered into the left articular cavity. Control animals received saline. Laser treatment started 14 days after MIA injection.

\section{CFA-induced inflammatory arthritis}

Articular damage was induced by injection of $50 \mu \mathrm{L}$ CFA (Sigma-Aldrich) into the tibiotarsal joint [15]. Briefly, the rats were slightly anaesthetized by $2 \%$ isoflurane, the left leg skin was sterilized with $75 \%$ ethyl alcohol, and the lateral malleolus located by palpation; then, a 28 -gauge needle was inserted vertically to penetrate the skin and turned distally for insertion into the articular cavity at the gap between the tibiofibular and tarsal bone until a distinct loss of resistance was felt. A volume of $50 \mu \mathrm{L}$ of CFA was then injected. Control animals received saline. Laser treatment started 14 days after CFA injection.

\section{CCI-induced peripheral mononeuropathy}

Neuropathy was induced according to the procedure described in Bennett and Xie [16]. Briefly, rats were anaesthetized with $2 \%$ isoflurane. Under aseptic conditions, the right (ipsilateral) common sciatic nerve was exposed at the level of the middle thigh by blunt dissection. Proximal to the trifurcation, the nerve was carefully freed from the surrounding connective tissue, and four chromic catgut ligatures (4-0, Ethicon, Norderstedt, Germany) were tied loosely around the nerve with about 1-mm spacing between ligatures. After hemostasis was confirmed, the incision was closed in layers. The animals were allowed to recover from surgery and then housed one per cage with free access to water and standard laboratory chow. 
Another group of rats were subjected to sham surgery in which the sciatic nerve was only exposed but not ligated. Laser treatment started 7 days after surgery.

\section{Laser treatments and study design (Fig. 1)}

MIA and CFA models were treated with a Multiwave Locked System laser (MLS-Mphi, ASA S.r.l., Vicenza, Italy). It is a class IV NIR laser with two synchronized sources (laser diodes). The first one is a pulsed laser diode, emitting at $905 \mathrm{~nm}$ wavelength, with $25 \mathrm{~W}$ peak optical power. The pulse frequency may be varied in the range 1-2000 Hz. The second laser diode emits at $808 \mathrm{~nm}$ wavelength and may operate in continuous ( $\max$ power $1 \mathrm{~W}$ ) or frequenced (repetition rate $1-2000 \mathrm{~Hz}, 50 \%$ duty cycle) mode. The two laser beams work simultaneously, synchronously and the propagation axes are coincident.

The treatments of the CCI model were performed with a new MLS-HPP experimental device designed to have the same kind of sources (laser diodes), emission wavelengths (808 $\mathrm{nm}$ and $905 \mathrm{~nm}$ ) and synchronized emission mode (continuous/frequenced and pulsed mode) of the MLS-Mphi laser, but with a significant increase in terms of power output. In the experimental device, different power settings can be chosen.

The pulsed source emits at $905 \mathrm{~nm}$ wavelength, with peak power from $140 \mathrm{~W}$ to $1 \mathrm{~kW} \pm 20 \%$. The pulse frequency may be varied in the range $1-2000 \mathrm{~Hz}(1-40 \mathrm{~Hz}$ for maximum power). The continuous source emits at $808 \mathrm{~nm}$ wavelength and may operate in continuous (max power $6 \mathrm{~W} \pm 20 \%$ ) or frequenced (repetition rate $1-2000 \mathrm{~Hz}, 50 \%$ duty cycle) mode. Also, the sources of the MLS-HPP experimental device work simultaneously and synchronously and the propagation axes are coincident.

In details:

\section{MIA group}

Ia. Control $(n=5)$. Animals injected with saline without MIA

Ib. MIA $(n=5)$, laser-untreated animals;

Ic. MIA-Laser-treated group $(n=5)$, animals exposed to laser radiation. The treatment started 14 days after MIA injection and was performed once a day for 13 days during 3 weeks (days $1-5 ; 8-12 ; 15-17$ ). It consisted in irradiation of two points, one located on the medial side of the patella and the other on lateral side. Each point was treated for $8 \mathrm{~s}$ with the following laser parameters: $30 \mathrm{~Hz}$, $25 \%$ int (mean power $125 \mathrm{~mW}$ ), peak power $90525 \mathrm{~W}$, $0.32 \mathrm{~J} / \mathrm{cm}^{2}, 1 \mathrm{~J} /$ point.

\section{CFA group}

IIa. Control $(n=5)$. Animals injected with saline instead of CFA
IIb. CFA $(n=5)$, laser-untreated animals;

IIc. CFA - Laser-treated group $(n=5)$, animals exposed to laser radiation. The treatment started 14 days after CFA injection and was performed once a day for 13 days during 3 weeks (days $1-5 ; 8-12 ; 15-17$ ). It consisted in irradiation of two points, as described in Ic. Each point was treated for $4 \mathrm{~s}$ with the following laser parameters: $30 \mathrm{~Hz}$ (mean power $125 \mathrm{~mW}$ ), peak power 905 $25 \mathrm{~W}, 25 \%$ int, $0.16 \mathrm{~J} / \mathrm{cm}^{2}, 0.5 \mathrm{~J} /$ point.

\section{CCI group}

IIIa. Sham $(n=5)$. Animal subjected to sham surgery in which the sciatic nerve was only exposed but not ligated.

IIIb. CCI $(n=5)$, laser-untreated animals;

IIIc. CCI - Laser-treated group $1(n=5)$, animals exposed to laser radiation. The treatment was started 7 days after surgery and was performed once a day for 10 days during 3 weeks (days 1-3; 6-10; 13-14). It consisted in irradiation of two points, one located directly on sciatic nerve ligation and the other on lateral side of the calcaneus (paw joint), followed by a scan of the limb performed by moving continuously the handpiece along the limb of the animal. Points were treated for $5 \mathrm{~s}$ with the following laser parameters: $30 \mathrm{~Hz}, 20 \%$ int (mean power $680 \mathrm{~mW}$ ), peak power ${ }_{905} 140 \mathrm{~W}, 1.08 \mathrm{~J} / \mathrm{cm}^{2}$, $3.4 \mathrm{~J} /$ point. Scan was performed for $124 \mathrm{~s}$ with the following parameters: $700 \mathrm{~Hz}, 50 \%$ int (mean power $1700 \mathrm{~mW}$ ), peak power $905140 \mathrm{~W}, 5.28 \mathrm{~J} / \mathrm{cm}^{2}, 211.13 \mathrm{~J}$.

IIId. CCI-Laser-treated group $2(n=5)$, animals exposed to laser radiation. The treatment was started 7 days after surgery and was performed once a day for 10 days during 3 weeks (days $1-3 ; 6-10 ; 13-14$ ) and consisted in irradiation of two points, followed by a scan of the limb (see IIIc). Points were treated for $3 \mathrm{~s}$ with the following laser parameters: $30 \mathrm{~Hz}, 20 \%$ int (mean power $980 \mathrm{~mW}$ ), peak power $9051 \mathrm{~kW} \pm 20 \%, 0.94 \mathrm{~J} / \mathrm{cm}^{2}, 2.96 \mathrm{~J} /$ point. Scan was performed for $108 \mathrm{~s}$ with the following parameters: $40 \mathrm{~Hz}, 50 \%$ int (mean power $1940 \mathrm{~mW}$ ), peak power $_{905} 1 \mathrm{~kW} \pm 20 \%, 5.24 \mathrm{~J} / \mathrm{cm}^{2}, 209.8 \mathrm{~J}$.

\section{Paw pressure test}

The nociceptive threshold in the rat was determined with an analgesimeter (Ugo Basile, Varese, Italy) according to the method described by Leighton et al. [17]. Briefly, a constantly increasing pressure was applied to a small area of the dorsal surface of the hind paw using a blunt conical mechanical probe. Mechanical pressure was increased until vocalization or a withdrawal reflex occurred while rats were lightly restrained. Vocalization or withdrawal reflex thresholds were 
Laser Treatments

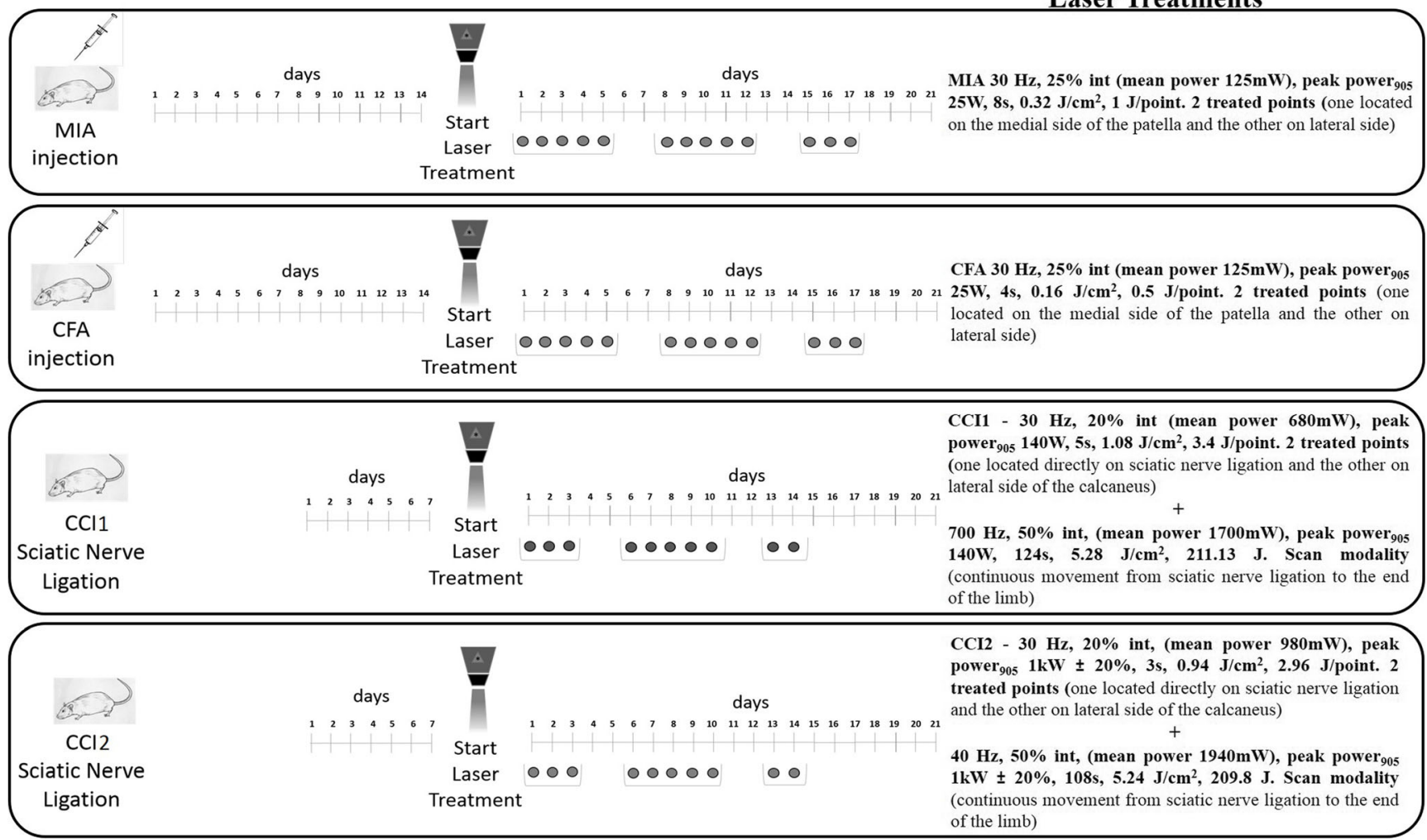

Fig. 1 Laser treatment protocols with time schedule and parameters used

expressed in grams. These limits assured a more precise determination of mechanical withdrawal threshold in experiments aimed to determine the effect of treatments. An arbitrary cut-off value of $100 \mathrm{~g}$ was adopted. For MIA and CFA, laser treatments were started 14 days after injury and Paw pressure test was performed on days $1-5$; 8-12; and 1517 at $0,5,30,60$ and $180 \mathrm{~min}$ after laser application. For CCI, laser treatments started 7 days after injury and Paw pressure test was performed on days $1-3 ; 6-10$; and 13-14 at 0,5 , 30, 60 and $180 \mathrm{~min}$ after laser application. The data were collected by an observer who was blinded to the protocol.

\section{Incapacitance test}

Weight bearing changes were measured using an incapacitance apparatus (Linton Instrumentation, UK) detecting changes in postural equilibrium after a hindlimb injury [18]. Rats were trained to stand on their hind paws in a box with an inclined plane $\left(65^{\circ}\right.$ from horizontal). This box was placed above the incapacitance apparatus. This allowed us to independently measure the weight that the animal applied on each hindlimb. The value considered for each animal was the mean of five consecutive measurements. In the absence of hindlimb injury, rats applied an equal weight on both hindlimbs, indicating a postural equilibrium, whereas an unequal distribution of the weight on hindlimbs indicated a monolateral decreased pain threshold.
Data are expressed as the difference between the weight applied on the limb contralateral to the injury and the weight applied on the ipsilateral one. This behavioural measurement was performed only on CCI rats on days $1-3 ; 6-10 ; 13-14$ at 0 and 60 min after laser application. Blind experiments were performed.

\section{Statistical analyses}

Behavioural measurements were performed on five rats for each treatment. Measurements were taken in duplicate at least $1 \mathrm{~min}$ apart; the responses of both left and right paws were measured. Results were expressed as mean (sem) with oneway analysis of variance (ANOVA). A Bonferroni's significant difference procedure was used as a post hoc comparison. Data were analysed using the "Origin 9.0" software (OriginLab, Northampton, MA, USA). Differences were considered significant at a $P<0.05$.

\section{Results}

\section{MIA-induced osteoarthritis}

The pain reliever effect of repeated laser treatments was evaluated on MIA-induced osteoarthritis model in the rat (in Fig. 2). 
Laser application started 2 weeks after MIA intra-articular injection (day-14), when the tolerance to a suprathreshold stimulation was reduced to $45.0 \pm 1.0 \mathrm{~g}$ in comparison to control animals $(62.5 \pm 1.4 \mathrm{~g})$ (day 1, 0 min; Fig. 2) and was performed once a day, for 13 days during 3 weeks (days $1-5$; $8-12 ; 15-17)$, using an MLS-Mphi laser. On the same days, the nociceptive threshold was measured by Paw pressure test before (black curve) and after ( $5 \mathrm{~min}$, blue curve; $30 \mathrm{~min}$, pink curve; $60 \mathrm{~min}$, green curve; and $180 \mathrm{~min}$, wine curve) treatment (Fig. 2). The first laser application increased of $24 \%$ the weight tolerated on the posterior paw $5 \mathrm{~min}$ after treatment. The anti-hypersensitivity effect remained significant after $30 \mathrm{~min}$ and vanished at $60 \mathrm{~min}(53.8 \pm 2.2$ and $43.3 \pm 0.8 \mathrm{~g}$, respectively). The pain reliever effect was no longer recorded after $24 \mathrm{~h}$ (day 2, before treatment), but new treatments (performed on days 2-5) were able to induce effects comparable to that obtained on day 1 . The four laser applications carried out from days 8 to 11 were less effective to reduce the nociceptive alteration induced by MIA than those performed previously. On the contrary, the following administrations (days $12,15-17)$ increased the pain threshold in a similarly (similar) to the first ones (days 1-5). The nociceptive threshold of the contralateral paw did not change during the entire duration of the experiment (data not shown). Also, the nociceptive threshold of the laser-untreated MIA group did not change significantly during the entire experiment, as shown in able 1.

\section{CFA-induced inflammatory arthritis}

In the CFA model, we evaluated the antinociceptive effect of repeated MLS-Mphi laser treatments in articular inflammatory damage resembling human rheumatoid arthritis (Fig. 3). In the same days of laser treatment, the hypersensitivity to a mechanical noxious stimulus (Paw pressure test) was measured before (black curve) and after (5 min, blue curve; $30 \mathrm{~min}$, pink curve; $60 \mathrm{~min}$, green curve and $180 \mathrm{~min}$, wine curve) treatment (Fig. 3). On day 1, before laser application, rats underwent to CFA intra-articular injection tolerated a weight of $43.0 \pm 0.8 \mathrm{~g}$ on the ipsilateral paw with respect to $62.5 \pm 1.4 \mathrm{~g}$ of the control group. Laser treatment reverted CFA-induced hypersensitivity 5 min after administration $(64.2 \pm 1.5 \mathrm{~g})$, the effect was still significant at $30(55.8 \pm 1.7 \mathrm{~g})$ and $60 \mathrm{~min}(50.0 \pm 0.3 \mathrm{~g})$ and disappeared at $180(44.2 \pm 0.8 \mathrm{~g})$. Although the pain reliever effect of laser was no longer recorded after $24 \mathrm{~h}$ (day 2, before treatment), a new laser application was able to reach a significant anti-hypersensitivity effect (at 5 and $30 \mathrm{~min}, 58.5 \pm 0.8$ and $55.3 \pm 1.5 \mathrm{~g}$, respectively) even if with a lower efficacy to that obtained on day 1 . Similar effects were observed the following days $(3-5,8-12$ and $15-17$; Fig. 3$)$. The nociceptive threshold of the contralateral paw did not changed during the entire duration of the experiment as well as the nociceptive threshold of the laser-untreated CFA group, shown in able 2.

\section{CCI-induced peripheral mononeuropathy}

After these results obtained irradiating directly the damage points (articular joint) in MIA and CFA models and following a preliminary study on the effect of a similar laser treatment in CCI-induced mononeuropathy, we carried out further experiments using a new MLS-HPP experimental device in order to evaluate its effectiveness in relieving CCI-induced mononeuropathy and comparing two setup with similar fluence but different output power. A first group of rats (IIIc) was treated using the setup characterized by lower power. Laser application started 1 week after surgery and was performed once a day (Fig. 4). The evaluation of hypersensitivity was carried out before (black curve) and after ( 5 min, blue curve; 30 min, pink curve; $60 \mathrm{~min}$, green curve; and $180 \mathrm{~min}$, wine curve) treatment on the ipsilateral (operated) paw. Sciatic nerve ligation reduced the pain response to a mechanical noxious stimulus (Paw pressure test; Fig. 4) from a value of $62.8 \pm 0.9 \mathrm{~g}$ in the sham animals to $46.3 \pm 0.7 \mathrm{~g}$ (day $1,0 \mathrm{~min}$ ), and this condition lingered until the end of the experiment (day 14). Laser application significantly increased the paw threshold at 5 and $30 \mathrm{~min}$ after treatment $(53.8 \pm 1.3$ and $51.9 \pm 1.2 \mathrm{~g}$, respectively). The anti-hypersensitivity effect was no longer recorded after $24 \mathrm{~h}$ (day 2, before treatment) but a new laser treatment evoked an effect comparable to that obtained in the previous day. Moreover, the effect was statistically significant after $60 \mathrm{~min}$ and vanished at $180 \mathrm{~min}$. Similar results were recorded during the following days of treatment (Fig. 4). Unilateral pain as CCI was also able to induce hindlimb weight bearing alterations (Incapacitance test; Fig. 4). The difference between the weight burdened on the contralateral and the ipsilateral paw was significantly increased in CCI rats (on day 1, 14 days after surgery) before laser treatment $(58.2 \pm 3.3 \mathrm{~g})$ with respect to the sham group $(5.2 \pm 3.6 \mathrm{~g})$. Laser administration reduced $50 \%$ of this gap at $60 \mathrm{~min}$. The relief was no longer showed $24 \mathrm{~h}$ later (Fig. 4). This trend was confirmed during the following administrations (days 2-3, 6-9, and 13-14).

The other group of rats (IIId) was treated with a similar protocol, but with the setup delivering higher power (Fig. 5).

On day 1 ( 7 days after injury), laser reverted the hypersensitivity induced by the sciatic nerve ligation at $5 \mathrm{~min}$ $(63.8 \pm 0.7$ vs $45.0 \pm 1.0 \mathrm{~g}$ recorded on $\min 0)$, the effect decreased at $30 \mathrm{~min}(57.5 \pm 1.0 \mathrm{~g})$ and disappeared at $60 \min (46.3 \pm 0.7 \mathrm{~g})$. During the following days of treatments, laser efficacy was effective also at $60 \mathrm{~min}$, vanishing $3 \mathrm{~h}$ later (Fig. 5).

As previously described, laser application was able to reduce of $50 \%$ the hindlimb weight bearing alteration induced by $\mathrm{CCI}$ at $60 \mathrm{~min}$ for the entire experiment (Fig. 5). able 3 lists the nociceptive threshold data for the CCI group (no laser treatment) measured by Paw pressure test during the entire experiment (Table 3): non-significant changes were monitored in the course of the experiment. 
Fig. 2 Osteoarthritis model induced by MIA. Intra-articular injection of MIA was performed 2 weeks before the beginning of the test (day-14). Laser treatment $\left(30 \mathrm{~Hz}, 25 \%\right.$ int, $8 \mathrm{~s}, 0.32 \mathrm{~J} / \mathrm{cm}^{2}$, $1 \mathrm{~J} /$ point, two treated points) was applied on days $1-5 ; 8-12$; and 15-17. The response to a noxious mechanical stimulus was measured by Paw pressure test.

Behavioural measurements were conducted before ( $0 \mathrm{~min}$, black curve) and after (5 min, blue curve; $30 \mathrm{~min}$, pink curve; $60 \mathrm{~min}$, green curve and $180 \mathrm{~min}$, wine curve) laser treatment. Each value represents the mean \pm sem of five rats per group. $* P<0.05$ and $* * P<0.01$ vs the values at $0 \mathrm{~min}$ on the same day

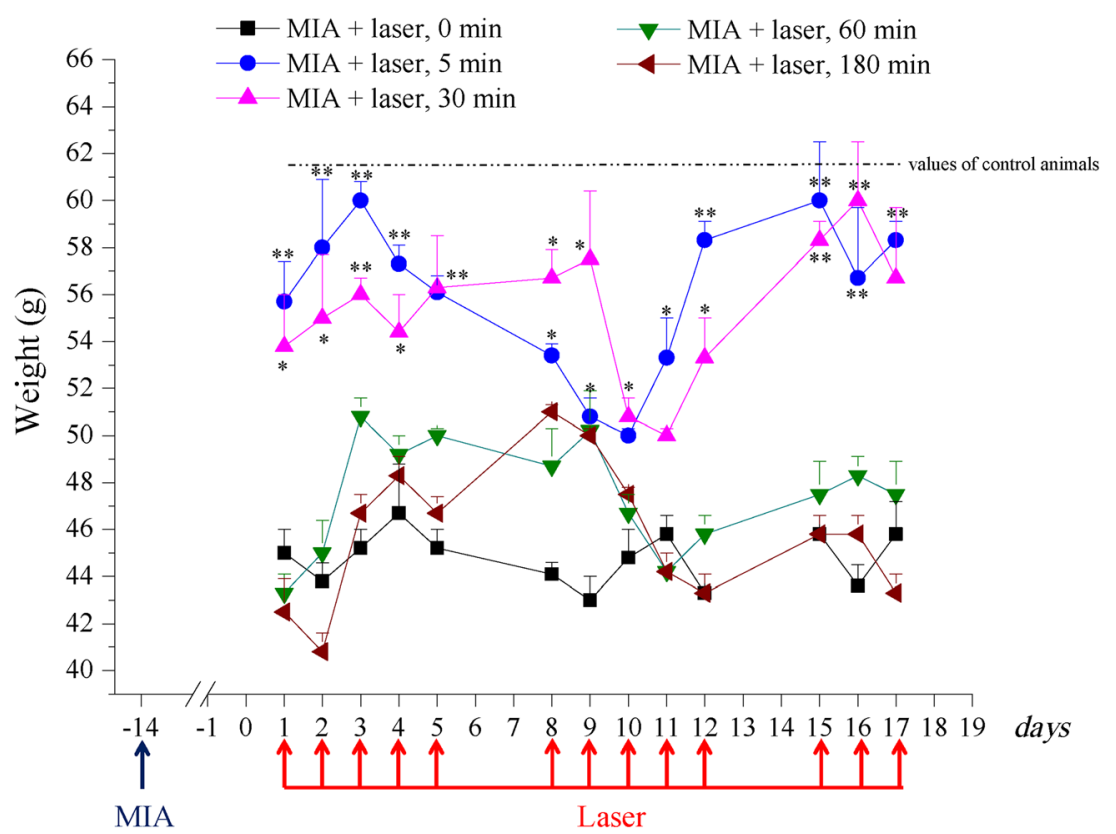

\section{Discussion}

The present results highlight the pain reliever effect of laser treatments in two rat models of articular pain (MIA and CFA) and in a rat model of CCI-induced mononeuropathy.

Rheumatoid arthritis (RA) and osteoarthritis (OA) are joint diseases characterized by different pathophysiological mechanisms, but displaying common clinical characteristics, such as joint pain, functional impairment and structural damage that are hallmarked by bone erosions in RA and osteophytes in
OA. Moreover, both diseases display joint space narrowing, reflecting cartilage loss [19].

$\mathrm{OA}$ is the most common subtype and it is particularly widespread among adults over age 60 years and those who are overweight or obese [20]. RA is an autoimmune disease involving extreme joint tissue inflammation [21]; it is far less pervasive and affects $1 \%$ of adults, typically with an onset at around age 40 years [22].

MIA-induced osteoarthritis in experimental animals is the best model to describe the effect of treatments on this
Table 1 Response to a mechanical noxious stimulus of MIA-treated animals, Paw pressure test

\begin{tabular}{|c|c|c|c|c|c|}
\hline \multirow[b]{2}{*}{ Days of laser treatment } & \multicolumn{5}{|l|}{ Weight $(g)$} \\
\hline & $0 \mathrm{~min}$ & $5 \mathrm{~min}$ & $30 \min$ & $60 \min$ & $180 \mathrm{~min}$ \\
\hline 1 & $45.0 \pm 1.2$ & $44.8 \pm 0.9$ & $46.3 \pm 1.0$ & $45.3 \pm 0.5$ & $42.7 \pm 2.2$ \\
\hline 2 & $43.9 \pm 2.4$ & $45.0 \pm 0.7$ & $44.6 \pm 1.3$ & $43.5 \pm 1.2$ & $46.7 \pm 0.3$ \\
\hline 3 & $45.7 \pm 0.7$ & $45.2 \pm 1.6$ & $43.1 \pm 2.6$ & $43.5 \pm 0.4$ & $45.8 \pm 0.6$ \\
\hline 4 & $44.8 \pm 0.4$ & $42.7 \pm 2.5$ & $44.8 \pm 0.8$ & $44.7 \pm 2.0$ & $44.6 \pm 1.4$ \\
\hline 5 & $45.6 \pm 1.3$ & $44.6 \pm 1.8$ & $43.6 \pm 1.2$ & $45.6 \pm 2.0$ & $45.0 \pm 0.9$ \\
\hline 8 & $43.6 \pm 1.2$ & $44.1 \pm 0.7$ & $45.7 \pm 1.1$ & $44.2 \pm 0.8$ & $44.0 \pm 1.2$ \\
\hline 9 & $43.6 \pm 0.8$ & $43.7 \pm 1.2$ & $44.6 \pm 1.4$ & $42.8 \pm 2.1$ & $44.6 \pm 1.4$ \\
\hline 10 & $44.1 \pm 0.7$ & $44.3 \pm 0.7$ & $42.8 \pm 1.6$ & $43.1 \pm 0.9$ & $44.5 \pm 0.6$ \\
\hline 11 & $45.6 \pm 0.4$ & $43.6 \pm 1.2$ & $44.6 \pm 0.9$ & $43.9 \pm 2.1$ & $44.1 \pm 1.5$ \\
\hline 12 & $43.8 \pm 0.8$ & $42.8 \pm 2.4$ & $43.8 \pm 1.1$ & $44.5 \pm 0.9$ & $43.8 \pm 1.2$ \\
\hline 15 & $44.6 \pm 1.0$ & $43.8 \pm 2.0$ & $46.7 \pm 0.5$ & $44.6 \pm 1.3$ & $44.1 \pm 0.8$ \\
\hline 16 & $45.7 \pm 3.0$ & $45.6 \pm 1.0$ & $43.7 \pm 1.6$ & $43.6 \pm 0.8$ & $45.8 \pm 0.9$ \\
\hline 17 & $46.2 \pm 0.9$ & $44.7 \pm 0.5$ & $46.7 \pm 0.7$ & $44.5 \pm 1.4$ & $43.7 \pm 1.3$ \\
\hline
\end{tabular}

Paw pressure test performed on MIA-treated animals. The response to a mechanical noxious stimulus was evaluated at the same time points of MIA-laser treated group. Behavioural measurements started 14 days after MIA intra-articular injection. Each value represents the mean \pm sem of five rats 
Fig. 3 Rheumatoid arthritis model induced by CFA. Intraarticular injection of CFA was performed 2 weeks before the beginning of the test (day-14). Laser treatment $(30 \mathrm{~Hz}, 25 \%$ int, $4 \mathrm{~s}, 0.16 \mathrm{~J} / \mathrm{cm}^{2}, 0.5 \mathrm{~J} /$ point, two treated points) was applied on days $1-5 ; 8-12$; and $15-17$. The response to a noxious mechanical stimulus was measured by Paw pressure test. Behavioural measurements were conducted before ( 0 min, black curve) and after ( $5 \mathrm{~min}$, blue curve; $30 \mathrm{~min}$, pink curve; $60 \mathrm{~min}$, green curve and $180 \mathrm{~min}$, wine curve) laser treatment. Each value represents the mean \pm sem of five rats per group. $* P<0.05$ and $* * P<0.01$ vs the values at $0 \mathrm{~min}$ on the same day

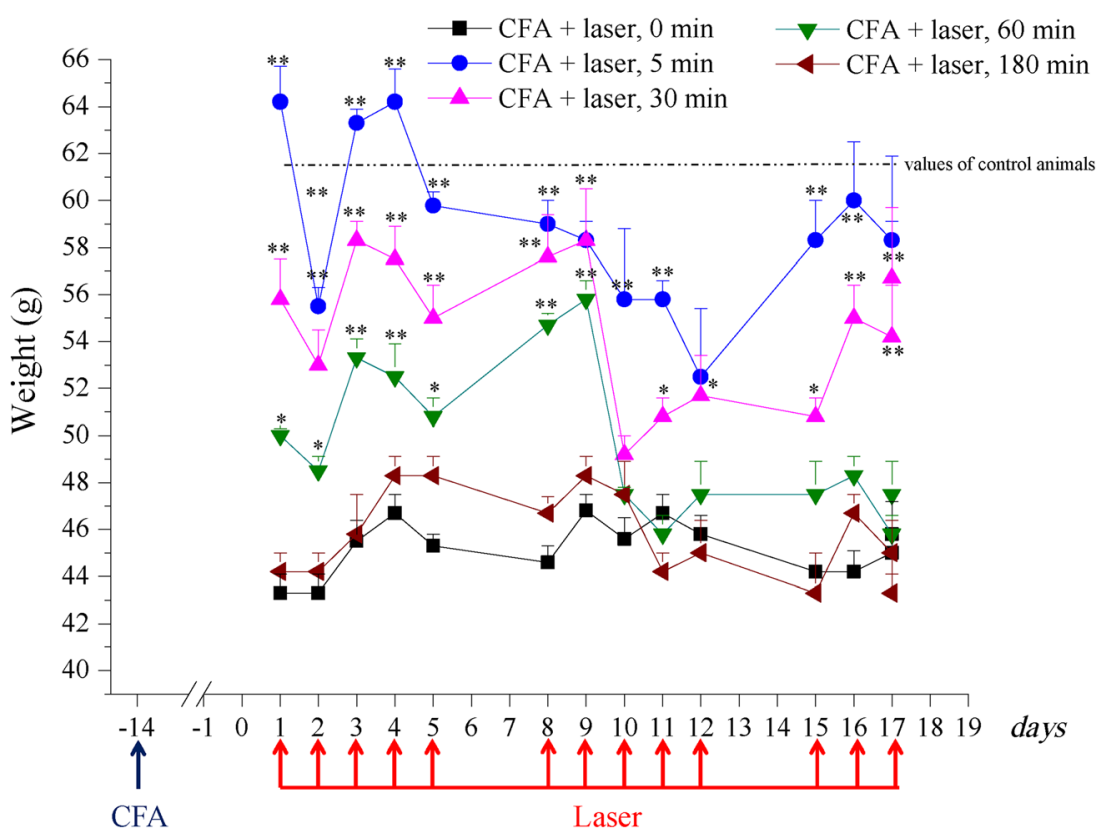

pathology because it mimics severe and acute OA pain that is similar with humans $[23,24]$. The histological and morphological alterations of the cartilage are associated with a persistent inflammatory pain which, starting from the 14th day after MIA injection, possesses a neuropathic component [14, 25]. The results of the experiments showed that daily laser treatments, consisting in the irradiation of two points, were able to increase the pain threshold of the animals from 5 to $30 \mathrm{~min}$ after laser application. Laser treatment started 14 days after MIA injection, when articular damages were established and rats showed a high degree of hypersensitivity against mechanical stimuli (Paw pressure test). Laser efficacy exhibited the same anti-hypersensitivity profile during the first week of treatment (days 1-5), while it decreased during the second week (days 8-12) to newly upturn on days 15-17. A similar protocol, with variation of time exposure and, consequently, dose administered, was used in a rat model of CFA-induced rheumatoid arthritis.

In the CFA arthritis model, the animal develops chronic swelling and pain in and around the joints with a release of
Table 2 Response to a mechanical noxious stimulus of CFA animals, Paw pressure test

\begin{tabular}{|c|c|c|c|c|c|}
\hline \multirow[b]{2}{*}{ Days of laser treatment } & \multicolumn{5}{|l|}{ Weight (g) } \\
\hline & $0 \mathrm{~min}$ & $5 \mathrm{~min}$ & $30 \mathrm{~min}$ & $60 \mathrm{~min}$ & $180 \mathrm{~min}$ \\
\hline 1 & $43.1 \pm 1.3$ & $42.4 \pm 1.5$ & $43.9 \pm 0.8$ & $42.8 \pm 1.4$ & $44.9 \pm 0.7$ \\
\hline 2 & $44.0 \pm 0.7$ & $43.7 \pm 0.9$ & $45.1 \pm 0.9$ & $44.4 \pm 0.8$ & $44.7 \pm 1.4$ \\
\hline 3 & $44.5 \pm 1.7$ & $42.8 \pm 2.0$ & $44.3 \pm 1.6$ & $44.8 \pm 0.7$ & $45.1 \pm 1.3$ \\
\hline 4 & $45.8 \pm 0.5$ & $44.7 \pm 1.0$ & $45.1 \pm 0.3$ & $45.3 \pm 0.6$ & $44.8 \pm 0.4$ \\
\hline 5 & $45.1 \pm 0.7$ & $43.7 \pm 0.8$ & $43.4 \pm 0.9$ & $45.6 \pm 1.4$ & $44.8 \pm 1.1$ \\
\hline 8 & $45.0 \pm 1.0$ & $45.7 \pm 0.4$ & $43.9 \pm 2.1$ & $44.6 \pm 1.3$ & $45.8 \pm 0.5$ \\
\hline 9 & $46.1 \pm 0.7$ & $45.4 \pm 1.0$ & $45.9 \pm 0.3$ & $45.7 \pm 1.3$ & $46.4 \pm 0.5$ \\
\hline 10 & $46.5 \pm 0.4$ & $45.8 \pm 2.0$ & $46.6 \pm 0.6$ & $44.2 \pm 2.1$ & $45.8 \pm 1.2$ \\
\hline 11 & $45.6 \pm 2.1$ & $46.1 \pm 0.9$ & $45.4 \pm 1.0$ & $46.3 \pm 0.9$ & $44.9 \pm 2.5$ \\
\hline 12 & $46.1 \pm 0.7$ & $46.2 \pm 0.3$ & $45.1 \pm 0.5$ & $46.1 \pm 0.4$ & $45.4 \pm 0.5$ \\
\hline 15 & $45.1 \pm 1.2$ & $45.9 \pm 0.9$ & $45.1 \pm 1.2$ & $46.3 \pm 0.8$ & $45.6 \pm 0.9$ \\
\hline 16 & $46.1 \pm 0.9$ & $46.2 \pm 0.4$ & $45.1 \pm 2.4$ & $46.1 \pm 0.9$ & $46.7 \pm 0.5$ \\
\hline 17 & $45.2 \pm 1.4$ & $45.9 \pm 0.7$ & $45.1 \pm 1.1$ & $46.3 \pm 0.6$ & $45.2 \pm 0.3$ \\
\hline
\end{tabular}

Paw pressure test performed on CFA-treated animals. The response to a mechanical noxious stimulus was evaluated at the same time points of CFA-laser treated group. Behavioural measurements started 14 days after CFA intra-articular injection. Each value represents the mean \pm sem of five rats 


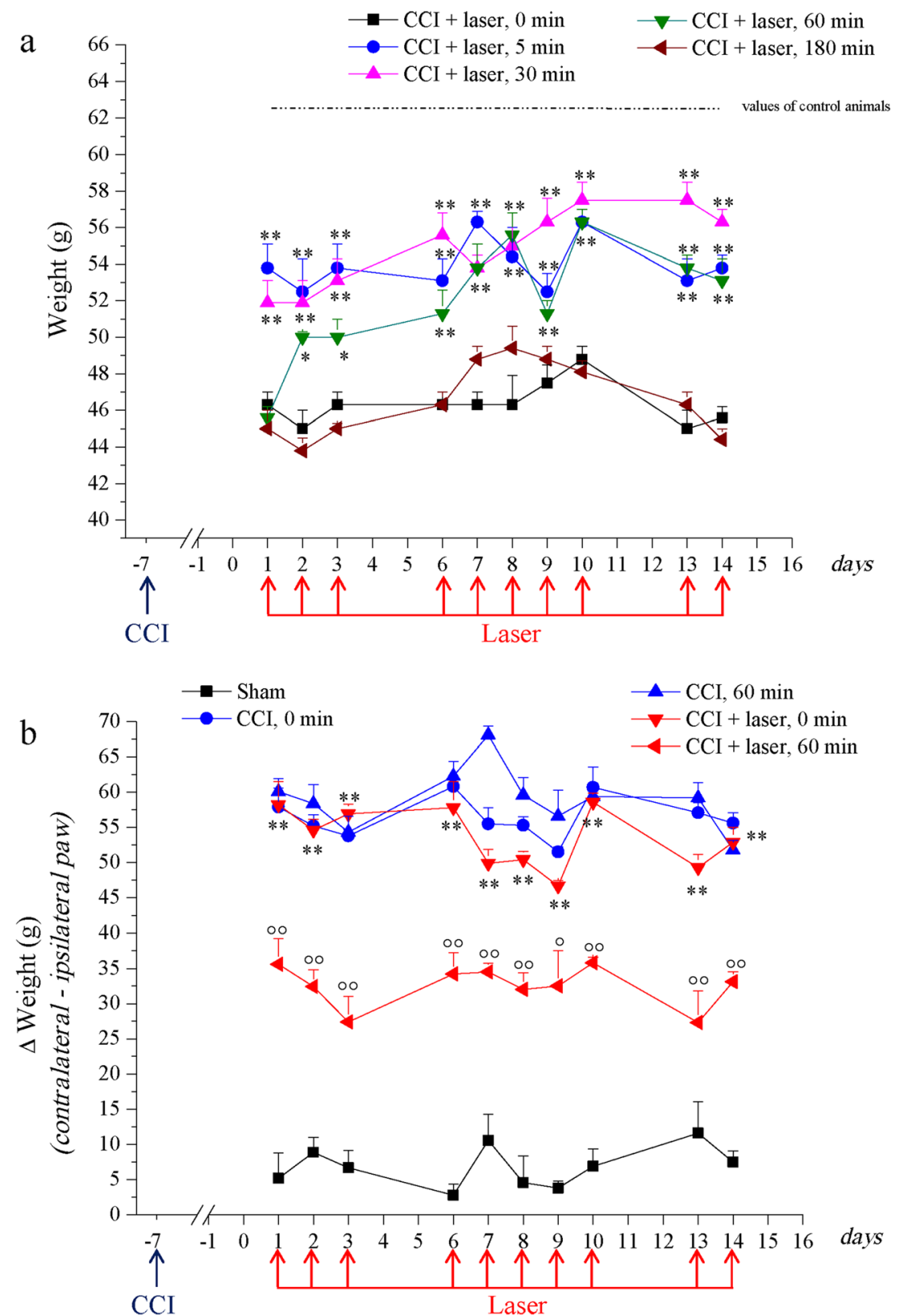

Fig. 4 a Monolateral neuropathy model induced by CCI. Sciatic nerve ligation was performed 7 days before the beginning of the test (day-7). Laser treatment [30 Hz, 20\% int (mean power $680 \mathrm{~mW}$ ), peak power 905 $140 \mathrm{~W}, 5 \mathrm{~s}, 1.08 \mathrm{~J} / \mathrm{cm}^{2}, 3.4 \mathrm{~J} /$ point, two treated points; scan $700 \mathrm{~Hz}, 50 \%$ int (mean power $1700 \mathrm{~mW}$ ), peak power ${ }_{905} 140 \mathrm{~W}, 124 \mathrm{~s}, 5.28 \mathrm{~J} / \mathrm{cm}^{2}$, $211.13 \mathrm{~J}$ tot] was applied on days $1-3 ; 6-10$; and $13-14$. The response to a noxious mechanical stimulus was measured by Paw pressure test. Behavioural measurements were conducted before ( 0 min, black curve) and after ( $5 \mathrm{~min}$, blue curve; $30 \mathrm{~min}$, pink curve; $60 \mathrm{~min}$, green curve and 180 min, wine curve) laser treatment. Each value represents the mean \pm sem of five rats per group. $* P<0.05$ and $* * P<0.01$ vs the

proinflammatory cytokines, which causes cartilage erosion and bone destruction. In this study, rats showed a progressive development of hypersensitivity to mechanical noxious stimulus (Paw pressure test) that peaked 14 days after CFA values at 0 min on the same day. b Monolateral neuropathy model induced by CCI. Sciatic nerve ligation was performed 7 days before the beginning of the test (day-7). Laser treatment $[30 \mathrm{~Hz}, 20 \%$ int (mean power $680 \mathrm{~mW}$ ), peak power $905140 \mathrm{~W}, 5 \mathrm{~s}, 1.08 \mathrm{~J} / \mathrm{cm}^{2}, 3.4 \mathrm{~J} /$ point, two treated points; scan $700 \mathrm{~Hz}, 50 \%$ int (mean power $1700 \mathrm{~mW}$ ), peak power $_{905} 140 \mathrm{~W}, 124 \mathrm{~s}, 5.28 \mathrm{~J} / \mathrm{cm}^{2}, 211.13 \mathrm{~J}$ tot] was applied on days 1-3; 6-10; and 13-14. The hindlimb weight bearing alteration was measured by Incapacitance test. Behavioural measurements were conducted before and $60 \mathrm{~min}$ after laser treatment. Each value represents the mean \pm sem of five rats per group. $* * P<0.01$ vs sham group; ${ }^{\circ} P<0.01 \mathrm{vs} \mathrm{CCI}+$ laser at 0 min on the same day

injection. At this time point, laser treatment started and was able to reduce the hypersensitivity from 5 to $60 \mathrm{~min}$ after treatment. In this model of articular damage, the efficacy of laser was higher during the first week of application and 


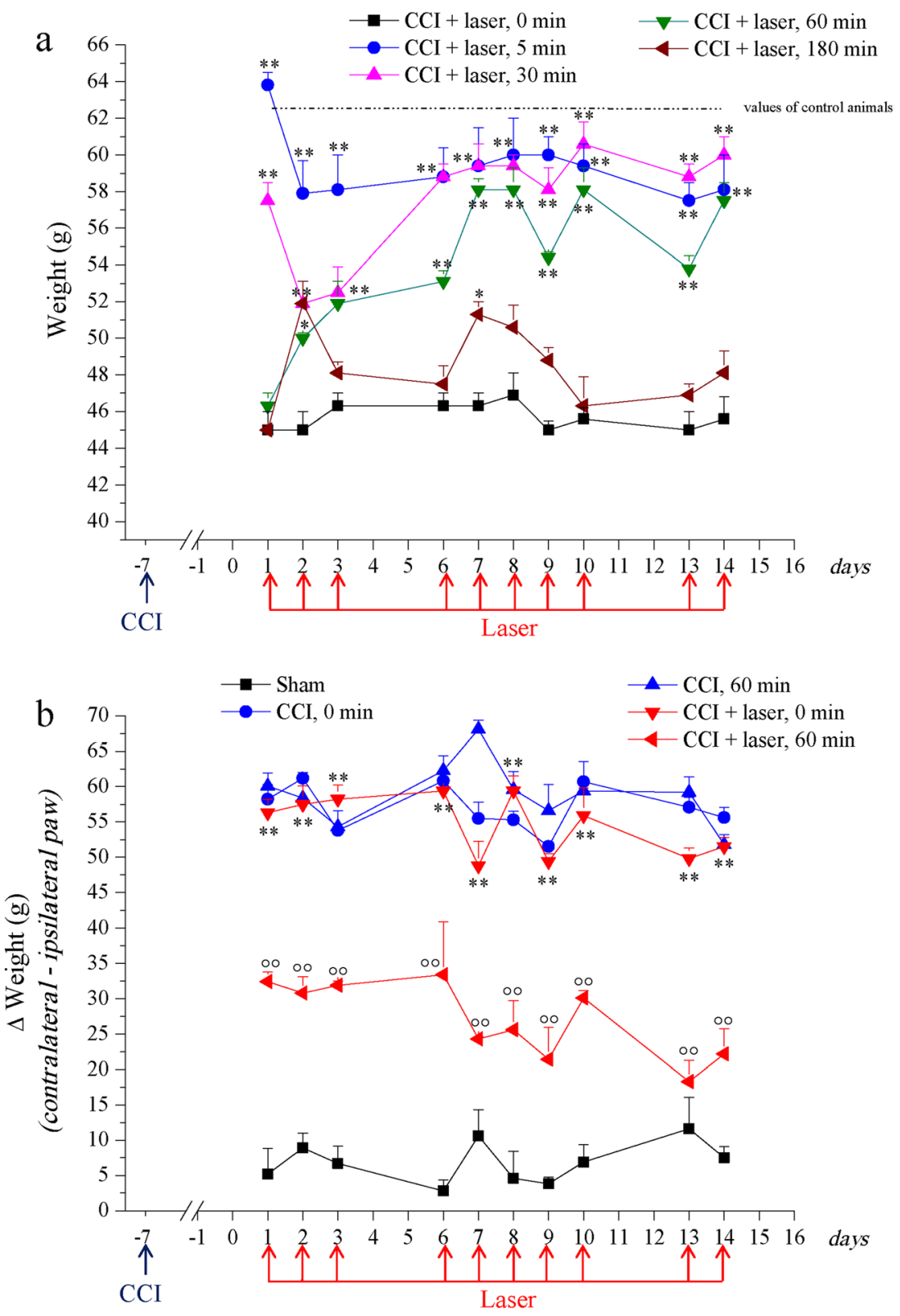

Fig. 5 a Monolateral neuropathy model induced by CCI. Sciatic nerve ligation was performed 7 days before the beginning of the test (day-7). Laser treatment $\left[30 \mathrm{~Hz}, 20 \%\right.$ int (mean power $980 \mathrm{~mW}$ ), peak power ${ }_{905}$ $1 \mathrm{~kW} \pm 20 \%, 3 \mathrm{~s}, 0.94 \mathrm{~J} / \mathrm{cm}^{2}, 2.96 \mathrm{~J} /$ point, two treated points; scan $40 \mathrm{~Hz}$, $50 \%$ int (mean power $1940 \mathrm{~mW}$ ), peak power ${ }_{905} 1 \mathrm{~kW} \pm 20 \%, 108 \mathrm{~s}$, $\left.5.24 \mathrm{~J} / \mathrm{cm}^{2}, 209.8 \mathrm{~J}\right]$ was applied on days $1-3 ; 6-10$; and 13-14. The response to a noxious mechanical stimulus was measured by Paw pressure test. Behavioural measurements were conducted before $(0 \mathrm{~min}$, black curve) and after ( $5 \mathrm{~min}$, blue curve; $30 \mathrm{~min}$, pink curve; $60 \mathrm{~min}$, green curve; and $180 \mathrm{~min}$, wine curve) laser treatment. Each value represents the mean \pm sem of five rats per group. $* P<0.05$ and $* * P<0.01$ vs the values at $0 \mathrm{~min}$ on the same day. $\mathbf{b}$. Monolateral

slightly decreased during the second and the third week. In both MIA- and CFA-treated rats, the anti-hypersensitivity effect of laser disappeared $24 \mathrm{~h}$ after treatment suggesting a symptomatic pain relief profile and this effect could be due to a temporary reduction of proinflammatory cytokines and, neuropathy model induced by CCI. Sciatic nerve ligation was performed 7 days before the beginning of the test (day-7). Laser treatment $\left[30 \mathrm{~Hz}, 20 \%\right.$ int (mean power $980 \mathrm{~mW}$ ), peak power ${ }_{905}$ $1 \mathrm{~kW} \pm 20 \%, 3 \mathrm{~s}, 0.94 \mathrm{~J} / \mathrm{cm}^{2}, 2.96 \mathrm{~J} /$ point, two treated points; scan $40 \mathrm{~Hz}, 50 \%$ int (mean power $1940 \mathrm{~mW}$ ), peak power ${ }_{905} 1 \mathrm{~kW} \pm 20 \%$, $108 \mathrm{~s}, 5.24 \mathrm{~J} / \mathrm{cm}^{2}, 209.8 \mathrm{~J}$ ] was applied on days 1-3; 6-10; and 13-14. The hindlimb weight bearing alteration was measured by Incapacitance test. Behavioural measurements were conducted before and $60 \mathrm{~min}$ after laser treatment. Each value represents the mean \pm sem of five rats per group. $* * P<0.01$ vs sham group ${ }^{\circ} P<0.05$ and ${ }^{\circ} P<0.01$ vs CCI + laser at $0 \mathrm{~min}$ on the same day

subsequently, to a decrease of inflammation. A previous study, carried out on a rat model of acute inflammation, demonstrated that $650 \mathrm{~nm}$ wavelength laser radiation strongly reduced $\mathrm{TNF} \alpha$ expression [26]. In a study subsequently performed on hemorrhagic lesions induced by immune complex in rat lungs, 
Table 3 Response to a mechanical noxious stimulus of CCI animals, Paw pressure test

\begin{tabular}{|c|c|c|c|c|c|}
\hline \multirow[b]{2}{*}{ Days of laser treatment } & \multicolumn{5}{|l|}{ Weight (g) } \\
\hline & $0 \mathrm{~min}$ & $5 \mathrm{~min}$ & $30 \mathrm{~min}$ & $60 \mathrm{~min}$ & $180 \mathrm{~min}$ \\
\hline 1 & $45.3 \pm 0.5$ & $44.5 \pm 1.1$ & $45.4 \pm 0.5$ & $44.9 \pm 1.2$ & $45.1 \pm 1.2$ \\
\hline 2 & $45.1 \pm 1.2$ & $45.6 \pm 0.9$ & $44.8 \pm 1.5$ & $45.6 \pm 0.4$ & $46.3 \pm 1.0$ \\
\hline 3 & $46.1 \pm 0.7$ & $46.2 \pm 1.0$ & $46.4 \pm 0.8$ & $45.4 \pm 1.1$ & $46.4 \pm 0.9$ \\
\hline 4 & $45.8 \pm 1.2$ & $46.3 \pm 0.5$ & $46.0 \pm 0.8$ & $45.8 \pm 1.2$ & $46.1 \pm 1.0$ \\
\hline 5 & $46.3 \pm 0.7$ & $46.4 \pm 0.9$ & $45.4 \pm 1.2$ & $46.8 \pm 1.4$ & $46.4 \pm 1.6$ \\
\hline 8 & $46.8 \pm 0.4$ & $46.1 \pm 0.8$ & $45.4 \pm 2.3$ & $46.0 \pm 1.7$ & $45.3 \pm 1.2$ \\
\hline 9 & $45.4 \pm 1.5$ & $46.2 \pm 0.3$ & $45.4 \pm 0.8$ & $46.8 \pm 0.5$ & $46.1 \pm 0.7$ \\
\hline 10 & $47.0 \pm 0.5$ & $46.3 \pm 1.2$ & $46.6 \pm 0.7$ & $46.7 \pm 0.2$ & $45.8 \pm 1.2$ \\
\hline 11 & $46.2 \pm 1.8$ & $45.3 \pm 0.4$ & $46.7 \pm 1.1$ & $44.9 \pm 1.2$ & $46.7 \pm 0.7$ \\
\hline 12 & $45.1 \pm 0.8$ & $46.8 \pm 0.7$ & $46.7 \pm 0.9$ & $45.3 \pm 1.3$ & $46.4 \pm 1.5$ \\
\hline
\end{tabular}

Paw pressure test performed on CCI-treated animals. The response to a mechanical noxious stimulus was evaluated at the same time points of CCI-laser treated group. Behavioural measurements started 7 days after CCI intraarticular injection. Each value represents the mean \pm sem of five rats the same authors observed that the anti-inflammatory effect elicited by $650 \mathrm{~nm}$ wavelength laser radiation was similar to that of pharmacological agents such as celecoxib and dexamethasone [26]. A significant reduction in PGE2 was also reported in a clinical study performed on patients with bilateral Achilles tendonitis [27]. Substances with hyperalgesic action, such as prostaglandins, are able to reduce the excitation threshold of nociceptive receptors. Therefore, the inhibition of their release can lead to pain reduction. This mechanism supports the hypothesis that laser application with suitable treatment parameters can improve pain symptoms by modulating inflammation and its mediators. Nevertheless, the reduction of proinflammatory cytokines may not be the only mechanism of action involved. Another mechanism that could be involved in lasermediated analgesia is based on opioid response and inhibition of neuronal transmission. It is well known that opioid peptides can inhibit sympathetic nervous system outflow, thus exerting analgesic action. Some studies demonstrated that laser radiation induces an increase in concentration of some opioids, concurrently with pain reduction reported by patients. Walker et al. [28], who first investigated this aspect, reported that laser radiation decreased pain and increased the urinary excretion of serotonin and its degradation products, such as 5-hydroxy indole acetic acid (5-HIAA). In a similar study, using $904 \mathrm{~nm}$ wavelength radiation to treat patients with chronic orofacial pain, Hansen et al.[29] observed that, although on average pain reduction was not statistically significant, there was an increase in the urinary excretion of 5HIAA in patients with reduced pain. A few years later, Laakso et al. [30] observed decreased pain and increased levels of adrenocorticotropic hormone and $\beta$-endorphin following laser irradiation of myofascial trigger points in patients affected by neck and shoulder pain.
Direct inhibition of neural activity has also been identified as a plausible mechanism of laser-mediated pain-relieving effect. In in vivo studies, it has been shown that laser irradiation can partially or totally inhibit nerve conduction and can affect conduction velocity and action potential amplitude [31]. Studies on rat models showed that laser radiation inhibits $\mathrm{A} \delta$ and $\mathrm{C}$ fibre transmission of nociceptive stimuli induced by cold/heat, chemicals and proinflammatory substances [32, 33]. Later studies demonstrated that laser irradiation can induce suppression of bradykinin evoked action potentials [34] and downregulation of kinin receptors [35, 36]. Moreover, a recent work demonstrated that laser treatment reduced mechanical hypersensitivity in a model of neuropathic pain induced by spinal nerve injury through the modulation of macrophage/microglial cells activation [37].

In this study, we tested also the efficacy of a new MLSHPP experimental device and compared two setups allowing treatments with similar dose but different power output in a model of mononeuropathy. This model evoked a pain syndrome characterized by increased response to a suprathreshold stimulation that begins about 3 days after nerve injury and reaches a plateau that lasts between 7 and 30 days [38]. In our experiments, we started the laser treatments 1 week after injury. Results highlighted the effectiveness of both protocols in alleviating pain, but a higher efficacy of the treatment performed with the higher power output was evident since the first day of application (complete reversion of hyperalgesia). Instead, no difference was recorded in the ability to reduce postural unbalance. As previously described for the experiments on MIA and CFA, the anti-hypersensitivity effect of laser applications was no longer recorded $24 \mathrm{~h}$ after treatment, suggesting a symptomatic pain relief. Conversely, the antinociceptive effect of laser remained stable during days without a loss of efficacy on the second and third week of 
treatment with both setups. This outcome could be due to the scan of the limb, administered in addition to the treatment on fixed points, which was not performed on arthritic rats, and/or to the higher power characterizing the new MLS-HPP experimental device. The efficacy of NIR laser treatment on rats underwent to CCI was already showed in our previous experiments. We demonstrated that the application of high power NIR laser (MLS-Mphi) statistically increased the pain threshold of the neuropathic animals [39]. These results are in agreement with a recent work showing that laser application (660 and $980 \mathrm{~nm}$ wavelength) increased thermal and mechanical threshold in the same model of CCI-induced neuropathy in the rat [40]. In conclusion, the results of this study demonstrate the efficacy of PBMT by high-power NIR laser in different models of articular pain and in a model of mononeuropathy in the rat. In MIA and CFA-treated animals, the antihypersensitivity effect was higher during the first week of treatment in comparison to the effect highlighted on the second and third week. On CCI-treated rats, the efficacy of laser application, performed using a new MLS-HPP experimental device, remained stable throughout the experiment. Moreover, in this model of mononeuropathy, the higher efficacy of the setup delivering higher power output was highlighted. These results lead to suggest a clinical application of these treatment protocols in patients with arthritis and neuropathic pain.

Acknowledgements The authors acknowledge ASA Srl for providing the laser sources used in this study.

Compliance with ethical standard All animal manipulations were carried out according to the Directive 2010/63/EU of the European parliament and of the European Union council (22 September 2010) on the protection of animals used for scientific purposes. Experiments involving animals have been reported according to ARRIVE guidelines.

Conflict of interest The authors declare that they have no conflict of interest.

Funding This research was funded by the Italian Ministry of Instruction, University and Research (MIUR) and by the University of Florence.

Ethical approval The ethical policy of the University of Florence complies with the Guide for the Care and Use of Laboratory Animals of the US National Institutes of Health (NIH Publication No. 85-23, revised 1996; University of Florence assurance number: A5278-01). Formal approval to conduct the experiments described was obtained from the Italian Ministry of Health (No. 54/2014-B) and from the Animal Subjects Review Board of the University of Florence.

Informed consent Informed consent was obtained from all individual participants included in the study.

\section{References}

1. Hall GC, Carroll D, Parry D, McQuay HJ (2006) Epidemiology and treatment of neuropathic pain: the UK primary care perspective. Pain 122:156-162

2. Voscopoulos C, Lema M (2010) When does acute pain become chronic? Br J Anaesth 105(Supp1):i69-i85

3. Woolf CJ (2004) Pain: moving from symptom control toward mechanism-specific pharmacologic management. Ann Intern Med 140:441-451

4. Langley P, Müller-Schwefe G, Nicolaou A, Liedgens H, Pergolizzi J, Varrassi G (2010) The impact of pain on labor force participation, absenteeism and presenteeism in the European Union. J Med Econ 13(4):662-672. doi:10.3111/13696998.2010.529379

5. Baron R, Binder A, Wasner G (2010) Neuropathic pain: diagnosis, pathophysiological mechanisms, and treatment. Lancet Neurol 9(8):807-819. doi:10.1016/S1474-4422(10)70143-5

6. Benyamin R, Trescot AM, Datta S, Buenaventura R, Adlaka R, Sehgal N, Glaser SE, Vallejo R (2008) Opioid complications and side effects. Pain Physician 11(2 Suppl):S105-S120

7. Attal N, Lanteri-Minet M, Laurent B, Fermanian J, Bouhassira D (2011) The specific disease burden of neuropathic pain: results of a French nationwide survey. Pain 152(12):2836-2843. doi:10.1016/j. pain.2011.09.014

8. Torrance N, Ferguson JA, Afolabi E, Bennett MI, Serpell MG, Dunn KM, Smith BH (2013) Neuropathic pain in the community: more under-treated than refractory? Pain 154:690-699

9. Chow RT, Barnsley LB, Heller GZ (2006) The effect of $300 \mathrm{~mW}$, $830 \mathrm{~nm}$ laser on chronic neck pain: a double-blind, randomized, placebo-controlled study. Pain 124:201-210

10. Enwemeka CS, Parker JC, Dowdy DC, Harkness EE, Sanford LE, Woodruff LD (2004) The efficacy of low-power lasers in tissue repair and pain control: a meta-analysis study. Photomed Laser Surg 22:323-329

11. Plog FMW (1980) Biophysical application of the laser beam. In: Koebner HK (ed) Lasers in medicine. Wiley, Chichester, pp 21-37

12. McGrath JC, Lilley E (2015) Implementing guidelines on reporting research using animals (ARRIVE etc.): new requirements for publication in BJP. Br J Pharmacol 172:3189-3193

13. Guingamp C, Gegout-Pottie P, Philippe L, Terlain B, Netter P, Gillet P (1997) Mono-iodoacetate-induced experimental osteoarthritis: a dose-response study of loss of mobility, morphology, and biochemistry. Arthritis Rheum 40(9):1670-1679

14. Di Cesare Mannelli L, Bani D, Bencini A, Brandi ML, Calosi L, Cantore M, Carossino AM, Ghelardini C, Valtancoli B, Failli P (2013) Therapeutic effects of the superoxide dismutase mimetic compound MnIIMe2DO2A on experimental articular pain in rats. Mediat Inflamm. doi:10.1155/2013/905360

15. Butler SH, Godefroy F, Besson JM, Weil-Fugazza J (1992) A limited arthritic model for chronic pain studies in the rat. Pain 48:73-81

16. Bennett GJ, Xie YK (1988) A peripheral mononeuropathy in rat that produces disorders of pain sensation those seen in man. Pain 33:87-107

17. Leighton G, Rodriguez R, Hill R, Hughes J (1988) Kappa-opioid agonists produce antinociception after i.v. and i.c.v. but not intrathecal administration in the rat. Br J Pharmacol 93(3):553-560

18. Bove SE, Calcaterra SL, Brooker RM, Huber CM, Guzman RE (2003) Weight bearing as a measure of disease progression and efficacy of anti-inflammatory compounds in a model of monosodium iodoacetate-induced osteoarthritis. Osteoarthr Cartil $11: 821-830$

19. Brouwers H, von Hegedus J, Toes R, Kloppenburg M, IoanFacsinay A (2015) Lipid mediators of inflammation in rheumatoid arthritis and osteoarthritis. Best Pract Res Clin Rheumatol 29(6): $741-755$ 
20. Garver MJ, Focht BC, Dials J, Rose M, Lucas AR, Devor ST, Emery CF, Hackshaw KV, Rejesky WJ (2014) Weight status and differences in mobility performance, pain symptoms, and physical activity in older, knee osteoarthritis patients. Arthritis. doi:10.1155/ 2014/375909

21. Chang X, Wei C (2011) Glycolysis and rheumatoid arthritis. Int J Rheum Dis 14:217-222

22. Lipsky PE (2005) Rheumatoid arthritis. In: Kasper DL, Braunwald E, Fauci AS, Hauser SL, Longo DL, Jameson JL (eds) Harrison's principles of internal medicine. McGraw Hill, New York, pp 19681977

23. Combe R, Bramwell S, Field MJ (2004) The monosodium iodoacetate model of osteoarthritis: a model of chronic nociceptive pain in rats? Neurosci Lett 370:236-240

24. Guzman RE, Evans MG, Bove S, Morenko B, Kilgore K (2003) Monoiodoacetate-induced histologic changes in subchondral bone and articular cartilage of rat femorotibial joints: an animal model of osteoarthritis. Toxicol Pathol 31:619-624

25. Ivanavicius SP, Ball AD, Heapy CG, Westwood FR, Murray F, Read SJ (2007) Structural pathology in a rodent model of osteoarthritis is associated with neuropathic pain: increased expression of ATF-3 and pharmacological characterization. Pain 128:272-282

26. Aimbire F, Albertini R, Pacheco MT, Castro-Faria-Neto HC, Leonardo PS, Iversen VV, Lopes-Martins RA, Bjordal JM (2006) Low-level laser therapy induces dose-dependent reduction of TNF $\alpha$ levels in acute inflammation. Photomed Laser Surg 24:33-37

27. Bjordal JM, Lopes-Martins RAB, Iversen VV (2006) A randomised, placebo controlled trial of low level laser therapy for activated Achilles tendinitis with microdialysis measurement of peritendinous prostaglandin E2 concentrations. Br J Sports Med 40:76-80

28. Walker J (1983) Relief from chronic pain by low-power laser irradiation. Neurosci Lett 43:339-344

29. Hansen HJ, Thorøe U (1990) Low power laser biostimulation of chronic orofacial pain. A double-blind placebo controlled crossover study in 40 patients. Pain 43(2):169-179

30. Laakso EL, Cramond T, Richardson C, Galligan JP (1994) Plasma ACTH and â-endorphin levels in response to low level laser therapy (LLLT) for myofascial trigger points. Laser Ther 6:133-142
31. Chow R, Armati P, Laakso EL, Bjordal JM, Baxter GD (2011) Inhibitory effects of laser irradiation on peripheral mammalian nerves and relevance to analgesic effects: a systematic review. Photomed Laser Surg 29(6):365-381. doi:10.1089/pho.2010.2928

32. Sato T, Kawatani M, Takeshige C, Matsumoto I (1994) Ga-Al-As laser irradiation neuronal activity associated with inflammation. Acupuncture Electro 19:141-151

33. Tsuchiya D, Kawatani M, Takeshige C (1994) Laser irradiation abates neuronal responses to nociceptive stimulation of rat-paw skin. Brain Res Bull 34:369-374

34. Jimbo K, Noda K, Suzuki H, Yoda K (1998) Suppressive effects of low-power laser irradiation on bradykinin evoked action potentials in cultured murine dorsal root ganglia cells. Neurosci Lett 240:9396

35. Bortone F, Santos H, Albertini R, Pesquero J, Costa M, Soiva J (2008) Low-level laser therapy modulates kinin receptors mRNA expression in the subplantar muscle of rat paw subjected to carrageenan-induced inflammation. Int Immunopharmacol 8:206210

36. Couture R, Harrisson M, Vianna R, Cloutier F (2001) Kinin receptors in pain and inflammation. Eur J Pharmacol 429:161-176

37. Kobiela Ketz A, Byrnes KR, Grunberg NE, Kasper CE, Osborne L, Pryor B, Tosini NL, Wu X, Anders JJ (2017) Characterization of macrophage/microglial activation and effect of photobiomodulation in the spinal nerve injury model of neuropathic pain. Pain Med 18(5):932-946

38. Pacini A, Di Cesare Mannelli L, Bonaccini L, Ronzoni S, Bartolini A, Ghelardini C (2010) Protective effect of alpha7 nAChR: behavioural and morphological features on neuropathy. Pain 150(3):542549

39. Di Cesare Mannelli L, Ghelardini C, Micheli L, Cialdai F, Vignali L, Fusi F, Monici M (2015) Effect of high power dual wavelength NIR laser emission in a rat model of compressive pain. Energy Health 14:12-17

40. Masoumipoor M, Jameie SB, Janzadeh A, Nasirinezhad F, Soleimani M, Kerdary M (2014) Effects of 660- and 980-nm low-level laser therapy on neuropathic pain relief following chronic constriction injury in rat sciatic nerve. Lasers Med Sci 29(5):15931598. doi:10.1007/s10103-014-1552-1 